\title{
A comunidade arbórea de um fragmento urbano de Floresta Atlântica após 40 anos de sucessão secundária (Juiz de Fora, Minas Gerais)
}

\author{
Breno Moreira \\ Fabrício Alvim Carvalho * \\ Universidade Federal de Juiz de Fora \\ Departamento de Botânica - ICB \\ Rua José Lourenço Kelmer, s/n, Campus Universitário \\ CEP 36036-900, Juiz de Fora - MG, Brasil \\ *Autor para correspondência \\ fabricio.alvim@gmail.com
}

Submetido em 04/10/2012

Aceito para publicação em 22/03/2013

\section{Resumo}

Neste estudo, objetivou-se avaliar a estrutura e a diversidade da comunidade arbórea de um fragmento urbano de floresta estacional semidecidual no campus da Universidade Federal de Juiz de Fora-MG. As árvores (DAP $\geq 5 \mathrm{~cm}$ ) foram amostradas em 15 parcelas aleatórias de $20 \times 20 \mathrm{~m}$. Ao todo, foram amostrados 920 indivíduos (1.533 ind.ha $\left.{ }^{-1}\right)$, distribuídos em 48 espécies, pertencentes a 24 famílias botânicas, dentre as quais Fabaceae (12 espécies), Euphorbiaceae (5 espécies) e Melastomataceae (4 espécies) e apresentaram maior riqueza. A comunidade apresentou forte dominância específica, com grande concentração dos valores de importância (VI) distribuídos nas cinco primeiras espécies (Pinus elliottii; Miconia latecrenata; Tibouchina granulosa; Peltphorum dubium; e Syzygium jambos), que, juntas, somaram 51,7\% do VI total. Das espécies mais importantes na comunidade, destaca-se a exótica $P$. elliottii, com VI $=15,9 \%$, sendo possível considerá-la uma invasora capaz de provocar considerável contaminação biológica no local. Como reflexo da forte dominância ecológica, o índice de diversidade de espécies de Shannon $\left(\mathrm{H}^{\prime}=2,84\right.$ nats.ind $\left.^{-1}\right)$ e a riqueza (48 espécies) foram baixos em comparação com florestas secundárias de mesma fitofisionomia da região. Este estudo possibilitou identificar os padrões gerais e particularidades da área, contribuindo para subsidiar a implementação e consolidação de práticas ambientais consistentes para a conservação da diversidade.

Palavras-chave: Diversidade arbórea; Espécies exóticas; Fitossociologia; Floresta secundária; Floresta urbana

\section{Abstract}

The tree community of an Atlantic Forest urban fragment after 40 years of secondary succession (Juiz de Fora, Minas Gerais, Brazil). In this study, one aimed to evaluate the structure and diversity of the tree community of an urban fragment of semideciduous seasonal forest in the campus of Universidade Federal de Juiz de Fora, Minas Gerais, Brazil. The trees $(\mathrm{DBH} \geq 5 \mathrm{~cm})$ were sampled in 15 random 20 x $20 \mathrm{~m}$ plots. Altogether, 920 individuals were sampled (1,533 ind.ha $\left.{ }^{-1}\right)$, distributed into 48 species, belonging to 24 botanical families, among which Fabaceae (12 species), Euphorbiaceae (5 species), and Melastomataceae (4 species), and they showed a greater richness. The community showed strong specific dominance, with a high concentration of importance values (IV) distributed into the first five species (Pinus elliottii; Miconia latecrenata; Tibouchina 
granulosa; Peltphorum dubium; and Syzygium jambos), which, together, accounted for 51.7\% of total IV. Among the most important species in the community, one highlights the exotic P. elliottii, with IV $=15.9 \%$, and it's possible to regard it as an invader able to cause significant biological contamination on site. As a reflection of the strong ecological dominance, the Shannon's index of species diversity $\left(\mathrm{H}^{\prime}=2.84\right.$ nats.ind-1) and the richness (48 species) were low when compared to secondary forests with the same phytophysiognomy from the region. This study allowed one to identify the general patterns and peculiarities in the area, contributing to support the implementation and consolidation of consistent environmental practices for the preservation of diversity.

Key words: Exotic species; Phytosociology; Secondary forest; Tree diversity; Urban forest

\section{Introdução}

Na microrregião de Juiz de Fora (Minas Gerais), grande parte da Floresta Atlântica sensu lato (OLIVEIRAFILHO; FONTES 2000) foi devastada para criação de pastagens, e atividades de cafeicultura e urbanização, e embora a cidade de Juiz de Fora ainda apresente manchas de floresta em regeneração em sua malha urbana, poucas são as Unidades de Conservação para conservação efetiva da cobertura florestal (PMJF, 2008). Esses fragmentos florestais urbanos representam ecossistemas valiosos para a melhoria da qualidade de vida nas cidades, possuindo diversas utilidades e fornecendo benefícios ambientais, sócio-culturais e econômicos, como por exemplo, abrigo para a fauna, desenvolvimento de processos ecológicos, estabilidade do micro-clima, interceptação de chuvas, proteção dos solos, manutenção das nascentes e melhoria da qualidade de vida local (ALVEY, 2006). A manutenção das florestas urbanas torna-se importante, pois essas áreas atuam reduzindo os índices de poeiras e alguns poluentes atmosféricos em suspensão, regulando a umidade e temperatura do ar, mantendo a permeabilidade, fertilidade e umidade do solo, protegendo-o contra erosão e reduzindo os níveis de ruídos, pois amortecem o barulho das cidades (ALVEY, 2006).

A Universidade Federal de Juiz de Fora (UFJF) destaca-se neste cenário por possuir fragmentos de Floresta Atlântica em seu campus. Originalmente, o terreno da UFJF era uma pastagem; com o objetivo de urbanizar a área, na década de 60 foi implantado o plantio de Pinus spp. e Eucalyptus spp. bordejando as pistas para os veículos e em sua parte central. Paralelamente, muitas áreas foram abandonadas e deixadas em processo de regeneração natural, possibilitando o desenvolvimento de fragmentos de florestas secundárias com idade atual em torno de 40 anos (I. Vieira, comunicação pessoal). Este é o caso do fragmento florestal do Instituto de Ciências Biológicas (ICB), alvo do presente estudo.

As florestas secundárias são comunidades resultantes de processos sucessionais após a perda da vegetação primária por causas antrópicas ou naturais. A composição e a estrutura arbórea de uma floresta secundária dependem de fatores como o histórico de uso da terra, condições climáticas, efeito de borda, distância de fontes de biodiversidade, bem como de eventos estocásticos (HALPERN, 1989). As florestas tropicais secundárias variam consideravelmente em relação à estrutura e diversidade, o que é decorrente de um conjunto de fatores, como intensidade, duração e frequência das perturbações (CHAZDON, 2008). As mudanças mais relevantes durante a sucessão em florestas tropicais são mudanças estruturais, como o aumento da altura do dossel, da densidade de árvores, da área basal e da biomassa aérea, e também mudanças na composição específica, o que ocorre quando a floresta passa gradativamente pela sua fase de construção (CHAZDON, 2008). Segundo Chazdon (2008), em florestas tropicais o incremento da área basal e da biomassa ocorre mais rapidamente nos primeiros 25 anos do processo de sucessão, sendo relacionado à elevada presença de espécies pioneiras e secundárias iniciais. No entanto, em algumas situações, mesmo após um longo período (60 a 100 anos), a floresta tropical pode não ser capaz de recuperar a área basal e volume existente nas florestas maduras, o que depende diretamente dos fatores que agem sobre a comunidade, especialmente das perturbações (CHAZDON, 2008).

Poucos foram os estudos desenvolvidos nas florestas da microrregião de Juiz de Fora, especialmente nos fragmentos urbanos, que exercem importantes funções 
ecológicas. Neste contexto, o objetivo deste trabalho é caracterizar a composição, diversidade florística e estrutura horizontal do componente arbóreo de um fragmento de floresta urbana em regeneração; além de evidenciar a importância de conservação destas áreas, buscando, assim, fornecer subsídios complementares para a compreensão da ecologia das florestas urbanas da Zona da Mata de Minas Gerais.

\section{Material e Métodos}

\section{Área de estudo}

A área de estudo está localizada no campus da Universidade Federal de Juiz de Fora (21 ${ }^{\circ} 43^{\prime}$ 'S, $43^{\circ} 22^{\prime}$ W), situada na Unidade Serrana da Zona da Mata, em altitude em torno de $850 \mathrm{~m}$, pertencente à Região Mantiqueira Setentrional, estado de Minas Gerais (ROCHA et al., 2003). A vegetação presente na área apresenta características de Floresta Estacional Semidecidual Montana (IBGE, 2012). Os solos da área são classificados como latossolos vermelho-amarelos e distróficos (ROCHA et al., 2003).

De acordo com a classificação de Köppen (1948), a região possui clima do tipo $\mathrm{Cwb}$, ou seja, clima mesotérmico com verões quentes e estação chuvosa também no verão e invernos frios e secos. Este clima pode ser definido, genericamente, como Tropical de Altitude, por corresponder a um tipo tropical influenciado pelos fatores altimétricos, em vista do relevo local apresentar altitudes médias entre 700 e $900 \mathrm{~m}$, que contribuem para a amenização da temperatura. Os índices pluviométricos anuais, obtidos pela Estação Climatológica Principal da UFJF $/ 5^{\circ}$ DISME (NÚMERO 83692), nas últimas décadas, apresentam médias próximas a $1.536 \mathrm{~mm}$. Por sua vez a média térmica anual oscila em torno de $18,9^{\circ} \mathrm{C}$ (CESAMA, 2010).

A área do campus da Universidade Federal de Juiz de Fora (UFJF) era ocupada por vegetação de pastagem até a década de 1960. Então, o projeto de paisagismo desenvolvido nesse campus culminou com a introdução de inúmeras espécies exóticas, como Pinus spp. e espécies lenhosas ornamentais. Merece destaque a espécie Pinus elliottii Engelm, que foi largamente utilizada no plantio junto às vias de acesso do campus, visando a um contraste com os traços firmes e rígidos das edificações. Desde então, a área está em processo de regeneração natural. Atualmente a área se encontra em estágio intermediário de sucessão secundária, com a presença de espécies arbóreas nativas e lianas formando um mosaico florestal por toda a extensão do campus da UFJF.

\section{Amostragem da vegetação}

A amostragem da vegetação seguiu o protocolo padrão adaptado das fitofisionomias florestais dos biomas Cerrado e Pantanal (FELFILI et al., 2005), que contempla as características estruturais das florestas estacionais neotropicais. Foi adotado o modelo de parcelas de área fixa, que tem como vantagem a padronização das unidades amostrais e a possibilidade de obter dados comparáveis com outros estudos desenvolvidos na região. A área total do fragmento florestal, de aproximadamente 1 ha, foi delimitada como universo amostral, sendo gradeada em parcelas de $20 \mathrm{x}$ $20 \mathrm{~m}$ para o sorteio das unidades amostrais. A realização de um censo não foi possível, pois embora o fragmento possua cerca de 1 ha, existem áreas de alta declividade, com dominância de espécies herbáceas. Através de um controle local, excluímos essas áreas e foram sorteadas e alocadas quinze parcelas de $20 \times 20 \mathrm{~m}$, totalizando uma área amostral de $6.000 \mathrm{~m}^{2}$ (0,6 ha).

No interior das parcelas foram amostrados todos os indivíduos arbóreos vivos e mortos em pé com diâmetro à altura do peito (DAP medido a $1,30 \mathrm{~m}$ acima do solo) maior ou igual a $5,0 \mathrm{~cm}$. Todos os indivíduos incluídos foram marcados com placas metálicas (marcação permanente), medidos quanto ao DAP e altura e identificados. O material botânico foi coletado com tesoura de alta poda e as amostras foram identificadas com auxílio de bibliografia taxonômica ampla e com materiais depositados no Herbário CESJ e no Laboratório de Ecologia Vegetal da UFJF, seguindo a circunscrição de famílias proposta por APG III (2009). 


\section{Análise dos dados}

A estrutura fitossociológica foi analisada em conformidade com Kent e Coker (1992), sendo calculados os parâmetros riqueza de espécies (S), densidades absoluta e relativa (DA e DR), frequências absoluta e relativa (FA e FR), dominâncias absoluta e relativa (DoA e DoR) e Valor de Importância (VI). Os cálculos foram realizados no software Microsoft Office Excel 2007®.

A diversidade de espécies foi analisada por meio do índice de diversidade de Shannon (H'), que considera a transformação logarítmica (logaritmo natural) da densidade das espécies, sendo mais influenciado pelas espécies de menor densidade ou "raras" na amostra (MAGURRAN, 2004). O índice de equabilidade de Pielou (J), baseado em H', foi utilizado para se estimar a uniformidade da comunidade, como forma de aferir o quão próximo a diversidade H' obtida estaria da diversidade H' hipotética máxima (MAGURRAN, 2004).

Análises de distribuição diamétrica foram realizadas tanto para a comunidade quanto para as populações de maior VI na comunidade, adotando-se o intervalo de classe de diâmetro de $5 \mathrm{~cm}$, conforme Carvalho e Nascimento (2009). Os gráficos foram elaborados no programa Microsoft Office Excel 2007®.

Para auxiliar nas interpretações ecológicas da comunidade, as espécies foram enquadradas em três grupos ecológicos: grupo status de conservação, grupo ecofisiológico e grupo de síndromes de dispersão. As espécies foram classificadas quanto ao seu grau de conservação de acordo com conceitos de Oliveira Filho e Scolforo (2008), sendo enquadradas em classes de frequência com base em 190 listagens florísticas no estado de Minas Gerais, sendo: A (Abundante) $=>60 \%$ de frequência nas listagens florísticas; $\mathrm{C}$ (Comum) $=>40 \%$ a $60 \%$; F (Frequente) $=>25 \%$ a $40 \%$; O $($ Ocasional $)=>15 \%$ a $25 \%$; R (Rara) $=>7,5 \%$ a $15 \%$; MR (Muito Rara) $=>2,5 \%$ a 7,5\%; RR (Raríssima) $=<2,5 \%$. Além destas, outra categoria, de espécies exóticas (EX), foi adicionada, tendo em vista a grande presença deste grupo na amostragem. A classificação em guildas de regeneração seguiu Oliveira-Filho e Scolforo
(2008), com as espécies classificadas de acordo com suas características ecológicas e sucessionais, tendo como principal fator de inclusão nas categorias, a quantidade de luz disponível para seu desenvolvimento, sendo: P (Pioneiras), SI (secundárias iniciais), ST (secundárias tardias), e CL (clímax). As espécies foram classificadas nas guildas de dispersão de acordo com van der Pijl (1982), nas categorias: anemocóricas (Ane), aquelas cujas sementes são disseminadas pelo vento; zoocóricas (Zoo), aquelas que apresentam características que indicam que a dispersão de sementes é feita por animais; e autocóricas (Aut), aquelas que dispersam suas sementes pela gravidade ou por deiscência explosiva.

\section{Resultados e Discussão}

\section{Composição florística e fitossociologia}

Foram amostrados 920 indivíduos (equivalente a 1533 ind.ha $^{-1}$ ), distribuídos em 48 espécies arbóreas, pertencentes a 24 famílias botânicas. Destas espécies, 40 foram identificadas em nível específico, permanecendo três identificadas até gênero, duas até família e quatro indeterminadas.

Dentre as famílias de maior riqueza específica estão Fabaceae (12 espécies), Euphorbiaceae (05) e Melastomataceae (04). Estes resultados, com predominância de espécies de Fabaceae e Melastomataceae, vão ao encontro dos padrões normalmente encontrados para as florestas estacionais semideciduais montanas de Minas Gerais (OLIVEIRAFILHO; SCOLFORO, 2008), mesmo se tratando de um fragmento florestal urbano, mostrando que apesar de perturbado este fragmento consegue manter um padrão florístico próximo aos observados em áreas naturais.

A Tabela 1 apresenta a estrutura fitossociológica do fragmento florestal, onde se observa uma comunidade com forte dominância específica, com grande concentração dos Valores de Importância (VI) nas cinco primeiras espécies (Pinus elliottii Engelm; Miconia latecrenata (DC.) Naudin, Tibouchina granulosa (Desr.) Cogn; Peltphorum dubium (Spreng.) Taub. e Syzygium jambos (L.) Alston), que juntas somaram 51,7\% do VI total.

Das espécies mais importantes na comunidade, 
pode-se destacar a exótica Pinus elliottii, que obteve o mais elevado VI $(15,9 \%)$ e uma expressiva dominância relativa ( $\mathrm{DoR}=27,89)$, podendo-se considerá-la como uma invasora capaz de provocar considerável contaminação biológica no local. Menon e Carvalho (2012), em estudo realizado em outra área do campus da UFJF, encontraram resultados semelhantes, com uma notória invasão da espécie $P$. elliottii, tanto em ambientes abertos quanto em ambientes colonizados por espécies nativas. Esta espécie apresenta distribuição fortemente agrupada no fragmento florestal, fato evidenciado pela sua baixa frequência relativa e concentração de muitos indivíduos em poucas parcelas. Em uma das parcelas analisadas, por exemplo, se concentra cerca de $50 \%$ (70 indivíduos) do total de indivíduos amostrados. Além disso, as parcelas onde essa espécie ocorre com maior densidade são justamente aquelas mais próximas das vias de circulação de veículos, onde a mesma foi inicialmente plantada para arborização. Portanto é visível que seus indivíduos se desenvolvem onde há maior disponibilidade de propágulos e condições ecológicas para isso.

TABELA 1: Parâmetros fitossociológicos das espécies arbóreas amostradas no fragmento florestal da Universidade Federal de Juiz de Fora, Minas Gerais, Brasil. Espécies ordenadas de forma decrescente segundo o Valor de Importância VI.

\begin{tabular}{|c|c|c|c|c|c|c|c|c|c|c|c|c|}
\hline Especie & Família & GE & GC & SD & DA & $\mathbf{A B}$ & FA & DR & DoR & FR & VI & $\begin{array}{l}\text { VI } \\
(\%)\end{array}$ \\
\hline Pinus elliottii Engelm* & Pinaceae & $\mathrm{Pi}$ & EX & Ane & 142 & 4,0055 & 9 & 15,43 & 27,89 & 4,37 & 47,70 & 15,90 \\
\hline Miconia latecrenata (DC.) Naudin & Melastomataceae & $\mathrm{Pi}$ & $\mathrm{F}$ & Zoo & 155 & 1,5291 & 15 & 16,85 & 10,65 & 7,28 & 34,78 & 11,59 \\
\hline Tibouchina granulosa (Desr.) Cogn. & Melastomataceae & $\mathrm{Si}$ & $\mathrm{C}$ & Ane & 133 & 1,1696 & 13 & 14,46 & 8,14 & 6,31 & 28,91 & 9,64 \\
\hline Peltophorum dubium (Spreng.) Taub. & Fabaceae & $\mathrm{Pi}$ & $\mathrm{O}$ & Aut & 69 & 1,5886 & 10 & 7,50 & 11,06 & 4,85 & 23,42 & 7,81 \\
\hline Syzygium jambos (L.) Alston* & Myrtaceae & $\mathrm{Si}$ & EX & Zoo & 89 & 0,5352 & 14 & 9,67 & 3,73 & 6,80 & 20,20 & 6,73 \\
\hline $\begin{array}{l}\text { Piptadenia gonoacantha (Mart.) J.F. } \\
\text { Macbr }\end{array}$ & Fabaceae & $\mathrm{Pi}$ & $\mathrm{RR}$ & Aut & 34 & 0,9913 & 11 & 3,70 & 6,90 & 5,34 & 15,94 & 5,31 \\
\hline $\begin{array}{l}\text { Senna macranthera (DC. ex Collad.) } \\
\text { H.S. Irwin \& Barneby }\end{array}$ & Fabaceae & $\mathrm{Pi}$ & $\mathrm{C}$ & Zoo & 31 & 0,3567 & 10 & 3,37 & 2,48 & 4,85 & 10,71 & 3,57 \\
\hline Cecropia glaziovii Snethl. & Urticaceae & $\mathrm{Pi}$ & $\mathrm{F}$ & Zoo & 23 & 0,3660 & 10 & 2,50 & 2,55 & 4,85 & 9,90 & 3,30 \\
\hline Piptocarpha macropoda (DC.) Baker & & $\mathrm{Pi}$ & $\mathrm{F}$ & Ane & 28 & 0,2418 & 9 & 3,04 & 1,68 & 4,37 & 9,10 & 3,03 \\
\hline $\begin{array}{l}\text { Dalbergia nigra (Vell.) Alemao ex } \\
\text { Benth. }\end{array}$ & Fabaceae & $\mathrm{Pi}$ & $\mathrm{O}$ & Ane & 34 & 0,2146 & 7 & 3,70 & 1,49 & 3,40 & 8,59 & 2,86 \\
\hline Croton floribundus Spreng. & Euphorbiaceae & $\mathrm{Pi}$ & MR & Aut & 16 & 0,1839 & 11 & 1,74 & 1,28 & 5,34 & 8,36 & 2,79 \\
\hline Luehea divaricata Mart. & Malvaceae & $\mathrm{Pi}$ & MR & Ane & 12 & 0,0543 & 9 & 1,30 & 0,38 & 4,37 & 6,05 & 2,02 \\
\hline Jacaranda micrantha Cham. & Bignoniaceae & $\mathrm{Si}$ & $\mathrm{R}$ & Ane & 12 & 0,3027 & 5 & 1,30 & 2,11 & 2,43 & 5,84 & 1,95 \\
\hline Miconia cinnamomifolia (DC.) Naudin & Melastomataceae & $\mathrm{Pi}$ & $\mathrm{F}$ & Zoo & 9 & 0,2593 & 5 & 0,98 & 1,81 & 2,43 & 5,21 & 1,74 \\
\hline $\begin{array}{l}\text { Schizolobium parahyba (Vell.) S.F. } \\
\text { Blake }\end{array}$ & Fabaceae & $\mathrm{Pi}$ & MR & Ane & 11 & 0,1673 & 5 & 1,20 & 1,16 & 2,43 & 4,79 & 1,60 \\
\hline Inga marginata Willd. & Fabaceae & $\mathrm{Pi}$ & $\mathrm{C}$ & Zoo & 2 & 0,5242 & 1 & 0,22 & 3,65 & 0,49 & 4,35 & 1,45 \\
\hline $\begin{array}{l}\text { Anadenanthera colubrina (Vell). } \\
\text { Brenan }\end{array}$ & Fabaceae & $\mathrm{Pi}$ & $\mathrm{O}$ & Ane & 12 & 0,1375 & 4 & 1,30 & 0,96 & 1,94 & 4,20 & 1,40 \\
\hline Sapium glandulosum (L.) Morong & Euphorbiaceae & $\mathrm{Si}$ & $\mathrm{C}$ & Zoo & 9 & 0,0402 & 4 & 0,98 & 0,28 & 1,94 & 3,20 & 1,07 \\
\hline Cupressus sempervirens L.* & Cupressaceae & $\mathrm{NC}$ & $\mathrm{EX}$ & $\mathrm{NC}$ & 5 & 0,2858 & 1 & 0,54 & 1,99 & 0,49 & 3,02 & 1,01 \\
\hline Eucaliptus sp. $1^{*}$ & Myrtaceae & $\mathrm{NC}$ & EX & Aut & 3 & 0,2789 & 1 & 0,33 & 1,94 & 0,49 & 2,75 & 0,92 \\
\hline Mangifera indica L.* & Anacardiaceae & $\mathrm{NC}$ & EX & Aut & 8 & 0,0432 & 3 & 0,87 & 0,30 & 1,46 & 2,63 & 0,88 \\
\hline
\end{tabular}




\begin{tabular}{|c|c|c|c|c|c|c|c|c|c|c|c|c|}
\hline Alchornea glandulosa Poepp. & Euphorbiaceae & $\mathrm{Pi}$ & $\mathrm{F}$ & Zoo & 4 & 0,0329 & 4 & 0,43 & 0,23 & 1,94 & 2,61 & 0,87 \\
\hline Myrcia tomentosa (Aubl.) DC. & Myrtaceae & $\mathrm{Pi}$ & $\mathrm{C}$ & Zoo & 5 & 0,0711 & 3 & 0,54 & 0,50 & 1,46 & 2,50 & 0,83 \\
\hline Casuarina equisetifolia $\mathrm{L} . *$ & Casuarinaceae & $\mathrm{NC}$ & EX & $\mathrm{NC}$ & 5 & 0,2002 & 1 & 0,54 & 1,39 & 0,49 & 2,42 & 0,81 \\
\hline Indet sp. 4 & Indeterminada & $\mathrm{NC}$ & $\mathrm{NC}$ & $\mathrm{NC}$ & 1 & 0,2493 & 1 & 0,11 & 1,74 & 0,49 & 2,33 & 0,78 \\
\hline Schinus terebinthifolius Raddi & Anacardiaceae & $\mathrm{Pi}$ & $\mathrm{C}$ & Zoo & 8 & 0,0626 & 2 & 0,87 & 0,44 & 0,97 & 2,28 & 0,76 \\
\hline $\begin{array}{l}\text { Alchornea triplinervia (Spreng.) Mull. } \\
\text { Arg. }\end{array}$ & Euphorbiaceae & $\mathrm{Pi}$ & $\mathrm{F}$ & Zoo & 6 & 0,0237 & 3 & 0,65 & 0,17 & 1,46 & 2,27 & 0,76 \\
\hline Miconia sp. 1 & Melastomataceae & $\mathrm{Pi}$ & $\mathrm{NC}$ & Zoo & 6 & 0,0189 & 3 & 0,65 & 0,13 & 1,46 & 2,24 & 0,75 \\
\hline Indet sp. 2 & Indeterminada & $\mathrm{NC}$ & $\mathrm{NC}$ & $\mathrm{NC}$ & 4 & 0,0827 & 2 & 0,43 & 0,58 & 0,97 & 1,98 & 0,66 \\
\hline Ficus sp. 1 & Moraceae & $\mathrm{NC}$ & $\mathrm{NC}$ & Zoo & 4 & 0,0602 & 2 & 0,43 & 0,42 & 0,97 & 1,83 & 0,61 \\
\hline $\begin{array}{l}\text { Campomanesia guazumifolia } \\
\text { (Cambess.) O Berg. }\end{array}$ & Myrtaceae & $\mathrm{Pi}$ & $\mathrm{F}$ & Zoo & 4 & 0,0275 & 2 & 0,43 & 0,19 & 0,97 & 1,60 & 0,53 \\
\hline $\begin{array}{l}\text { Myrsine coriacea (Sw.) R. Br. ex } \\
\text { Roem. \& Schult. }\end{array}$ & Myrsinaceae & $\mathrm{Pi}$ & $\mathrm{R}$ & Zoo & 3 & 0,0337 & 2 & 0,33 & 0,23 & 0,97 & 1,53 & 0,51 \\
\hline Euphorbia cotinifolia L.* & Euphorbiaceae & $\mathrm{NC}$ & EX & $\mathrm{NC}$ & 4 & 0,0124 & 2 & 0,43 & 0,09 & 0,97 & 1,49 & 0,50 \\
\hline Machaerium nictitans (Vell.) Benth. & Fabaceae & $\mathrm{Pi}$ & $\mathrm{C}$ & Ane & 2 & 0,0348 & 2 & 0,22 & 0,24 & 0,97 & 1,43 & 0,48 \\
\hline $\begin{array}{l}\text { Tabebuia chrysotricha (Mart ex A. } \\
\text { DC.) Standl. }\end{array}$ & Bignoniaceae & $\mathrm{Pi}$ & $\mathrm{R}$ & Ane & 3 & 0,0167 & 2 & 0,33 & 0,12 & 0,97 & 1,41 & 0,47 \\
\hline Zanthoxylum rhoifolium Lam. & Rutaceae & $\mathrm{Pi}$ & $\mathrm{R}$ & Zoo & 2 & 0,0241 & 2 & 0,22 & 0,17 & 0,97 & 1,36 & 0,45 \\
\hline Cyathea sp. 1 & Cyathaceae & $\mathrm{NC}$ & $\mathrm{F}$ & Ane & 4 & 0,0387 & 1 & 0,43 & 0,27 & 0,49 & 1,19 & 0,40 \\
\hline Licania hoehnei Pilg. & Chrysobalanaceae & $\mathrm{NC}$ & $\mathrm{R}$ & $\mathrm{NC}$ & 2 & 0,0146 & 1 & 0,22 & 0,10 & 0,49 & 0,80 & 0,27 \\
\hline Citharexylum myrianthum Cham. & Verbenaceae & $\mathrm{Si}$ & $\mathrm{R}$ & Zoo & 2 & 0,0111 & 1 & 0,22 & 0,08 & 0,49 & 0,78 & 0,26 \\
\hline Vismia guianensis (Aubl.) Pers. & Clusiaceae & $\mathrm{Pi}$ & MR & Zoo & 2 & 0,0089 & 1 & 0,22 & 0,06 & 0,49 & 0,76 & 0,25 \\
\hline Indet sp. 1 & Indeterminada & $\mathrm{NC}$ & $\mathrm{NC}$ & $\mathrm{NC}$ & 1 & 0,0106 & 1 & 0,11 & 0,07 & 0,49 & 0,67 & 0,22 \\
\hline Indet sp. 3 & Indeterminada & $\mathrm{NC}$ & $\mathrm{NC}$ & $\mathrm{NC}$ & 1 & 0,0088 & 1 & 0,11 & 0,06 & 0,49 & 0,66 & 0,22 \\
\hline Cupania oblongifolia Mart. & Sapindaceae & $\mathrm{Pi}$ & $\mathrm{R}$ & Zoo & 1 & 0,0087 & 1 & 0,11 & 0,06 & 0,49 & 0,65 & 0,22 \\
\hline Cupania ludowigii Sommer \& Ferrucci & Sapindaceae & $\mathrm{Pi}$ & $\mathrm{O}$ & Zoo & 1 & 0,0058 & 1 & 0,11 & 0,04 & 0,49 & 0,63 & 0,21 \\
\hline $\begin{array}{l}\text { Enterolobium contortisiliquum (Vell.) } \\
\text { Morong }\end{array}$ & Fabaceae & $\mathrm{Pi}$ & $\mathrm{F}$ & Zoo & 1 & 0,0058 & 1 & 0,11 & 0,04 & 0,49 & 0,63 & 0,21 \\
\hline Lauraceae sp. 1 & Lauraceae & $\mathrm{NC}$ & $\mathrm{NC}$ & Zoo & 1 & 0,0058 & 1 & 0,11 & 0,04 & 0,49 & 0,63 & 0,21 \\
\hline Piperaceae sp. 1 & Piperaceae & $\mathrm{Si}$ & $\mathrm{NC}$ & $\mathrm{NC}$ & 1 & 0,0031 & 1 & 0,11 & 0,02 & 0,49 & 0,62 & 0,21 \\
\hline Erythrina speciosa Andrews & Fabaceae & $\mathrm{Pi}$ & $\mathrm{RR}$ & $\mathrm{NC}$ & 1 & 0,0030 & 1 & 0,11 & 0,02 & 0,49 & 0,62 & 0,21 \\
\hline Psychotria vellosiana Benth. & Rubiaceae & $\mathrm{Si}$ & $\mathrm{F}$ & Zoo & 1 & 0,0027 & 1 & 0,11 & 0,02 & 0,49 & 0,61 & 0,20 \\
\hline Bauhinia forficata Link. & Fabaceae & $\mathrm{Pi}$ & $\mathrm{F}$ & Aut & 1 & 0,0022 & 1 & 0,11 & 0,02 & 0,49 & 0,61 & 0,20 \\
\hline Apuleia leiocarpa (Vogel) J.F. Macbr. & Fabaceae & St & $\mathrm{F}$ & Ane & 1 & 0,0020 & 1 & 0,11 & 0,01 & 0,49 & 0,61 & 0,20 \\
\hline Eriobotrya japonica (Thunb.) Lindl.* & Rosaceae & $\mathrm{NC}$ & EX & Zoo & 1 & 0,0020 & 1 & 0,11 & 0,01 & 0,49 & 0,61 & 0,20 \\
\hline
\end{tabular}

Siglas: GE: grupo ecofisiológico (PI: pioneira; SI: secundária inicial; ST: secundária tardia; NC: não classificada); GC: grupo de status de conservação (A: abundante; C: comum; F: frequente; O: ocasional; R: rara; MR: muito rara; RR: raríssima); SD: síndrome de dispersão (Zoo: zoocórica; Ane: anemocórica; Aut: autocórica); DA: densidade absoluta (.ha-1); AB: área basal $\left(\mathrm{m}^{2} \cdot \mathrm{ha}^{-1}\right)$; DR: densidade relativa; DoR: dominância relativa; FR: frequência relativa; VI: Valor de Importância; *Espécies exóticas. 
A espécie pioneira Miconia latecrenata, segunda maior em VI (11,59\%), destacou-se pela elevada densidade absoluta (DA) e frequência absoluta (FA), sendo a espécie com maior número de indivíduos (155 indivíduos), representando $17 \%$ do total de indivíduos e ocorrendo em todas as parcelas analis/adas. Estes parâmetros (DA e FA) também foram elevados para outra pioneira, Tibouchina granulosa, terceira maior em VI $(9,64 \%)$. Esta espécie apresentou o terceiro maior número de indivíduos (133 indivíduos), representando aproximadamente $14 \%$ do total, ocorrendo em 13 das 15 parcelas analisadas. O fato da espécie Peltophorum dubium ter sido a quarta em VI $(7,81 \%)$ no fragmento resulta do grande porte dos indivíduos, obtendo o segundo maior valor de dominância relativa - DoR $(11,06)$. A elevada densidade desta espécie no fragmento provavelmente justifica-se pela sua grande utilização na ornamentação e paisagismo, bem como devido às condições históricas do local, que permitiram sua instalação e desenvolvimento (LORENZI, 1992). A proximidade com fontes de propágulos oriundos de áreas adjacentes contribuiu para a disseminação desta espécie no local, visto que em outros estudos desenvolvidos na região ela não ocorre com grande frequência (GARCIA, 2007).

Outra espécie exótica com grande representatividade na comunidade foi Syzygium jambos. Esta apresentou o quinto maior VI $(6,73 \%)$, principalmente pela alta densidade relativa na comunidade. $S$. jambos é uma espécie de origem asiática que possui forte caráter invasor, sendo apontada como uma invasora de potencial elevado em florestas neotropicais perturbadas (KUEFFER, 2010).

Estes resultados vêm corroborar a elevada representatividade de espécies exóticas no fragmento (15,4\% do total de espécies e $28 \%$ do total de indivíduos). Essa característica é bastante peculiar à área investigada, visto que este elevado percentual de espécies exóticas não havia sido encontrado em outros estudos em florestas semideciduais da região (ALMEIDA; SOUZA, 1997; GARCIA, 2007).

O valor da área basal obtida para os indivíduos vivos da comunidade foi $24,1 \mathrm{~m}^{2}$.ha $\mathrm{h}^{-1}$, próximo aos descritos para florestas estacionais secundárias na região (MARANGON et al., 2007), mostrando que a comunidade está sendo capaz de avançar em sua fase de construção, inicial ("early building phase") em termos de acúmulo de biomassa, expresso pelo acúmulo de área basal (OLDEMAN, 1983). Este valor sofreu grande influência das espécies $P$. elliottii e $P$. dubium, que são espécies de crescimento rápido e que apresentam elevado número de indivíduos de grande porte no fragmento. Com o aumento da idade da floresta a área basal tende a aumentar, fenômeno visto como uma das principais características da sucessão em florestas tropicais (CHAZDON, 2008).

Foram registradas 83 árvores mortas em pé, que em porcentagem correspondem a $8,3 \%$ dos indivíduos, com sua grande maioria (76\%) inclusa na primeira classe de diâmetro (Figura 1), representada pelos indivíduos mais jovens e que são justamente os mais susceptíveis à mortalidade na competição por recursos (CHAZDON, 2008). Este valor, considerado alto em termos de florestas neotropicais, está em conformidade com o padrão típico para florestas neotropicais secundárias (CHAZDON, 2008).

\section{Diversidade}

O valor do índice de Shannon (H') foi de 2,84 nats. ind $^{-1}$, e o de equabilidade de Pielou (J') foi de 0,72. O valor de $\mathrm{H}^{\prime}$ foi baixo em comparação aos encontrados

FIGURA 1: Distribuição dos indivíduos arbóreos mortos em pé por classes de diâmetro.

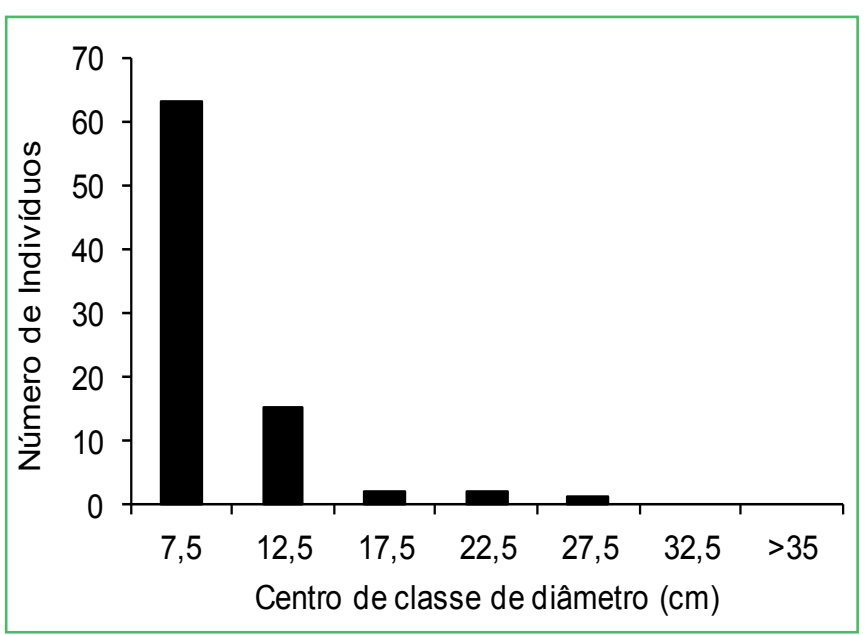


em outros fragmentos florestais de mesma fitofisionomia, e com diferentes históricos de perturbação no domínio da Floresta Atlântica do Sudeste de Minas Gerais, com cronosequências semelhantes de regeneração natural, onde em geral esse índice varia entre 3,00 e 3,50 nats. ind $^{-1}$ (GONZAGA et al., 2008).

Complementarmente, o valor de J' encontrado no presente estudo mostra que apenas $72 \%$ da diversidade (H') hipotética máxima foi obtida, sendo reflexo da forte dominância exercida por um pequeno conjunto de espécies na comunidade. De acordo com RondonNeto et al. (2000), a regeneração de uma floresta se desenvolve de um estado menos complexo para uma forma de maior complexidade, sendo esta influenciada pelo tempo, frequência e tipos de distúrbios do local.
Há de se considerar que a localização da área de estudo, junto a uma região do campus de intensa atividade humana, exerce grande influência sobre o processo de regeneração que mesmo após 40 anos de sucessão ainda apresenta características típicas de uma área em estágio intermediário de regeneração.

\section{Distribuição diamétrica}

A comunidade estudada apresentou padrão típico de J-reverso (Figura 2A), com maior percentagem de indivíduos (62,7\% do total), concentrando-se na menor classe de diâmetro (DAP entre 5 e $10 \mathrm{~cm}$ ) e com percentual de indivíduos com maiores DAP decaindo progressivamente, até a baixa percentagem de indivíduos com DAP maior que $35 \mathrm{~cm}(1,3 \%$ do total de indivíduos

FIGURA 2: Distribuição dos indivíduos arbóreos vivos (A) e das espécies Miconia latecrenata (B), Pinus elliottii (C) e Syzygium jambos (D) por classes de diâmetro.

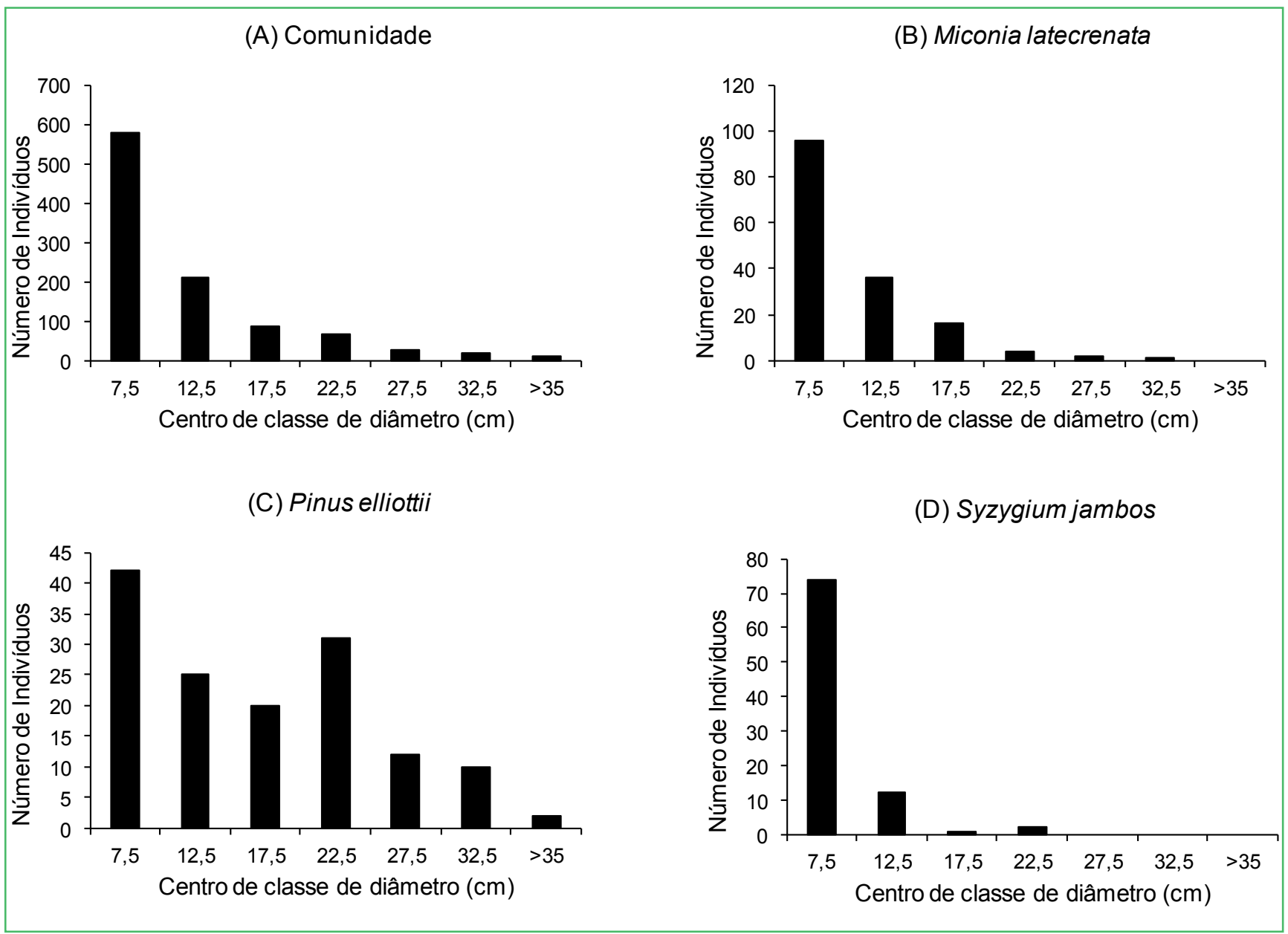


vivos). Este padrão também foi encontrado para as espécies nativas mais representativas da comunidade (Figura 2B)

Segundo Harper (1990), o padrão de J-invertido é encontrado em comunidades estáveis e autorregenerativas, em que existe um balanço entre mortalidade e o recrutamento dos indivíduos, pois o estoque de jovens será capaz de substituir os adultos senis ou em decrepitude. O alto percentual de árvores finas, inclusas na primeira classe de diâmetro (Figura $2 \mathrm{~A}$ ), pode indicar que o fragmento encontra-se em um estágio mais inicial de regeneração, pois segundo Uhl e Murphy (1981), florestas em estágios mais iniciais de regeneração apresentam grandes adensamentos de árvores finas, quando comparados com florestas maduras, que apresentam um elevado número de indivíduos com áreas basais grandes.

Dentre as espécies mais importantes na comunidade, a única população que não apresentou padrão J-reverso tão nítido foi P. elliottii (Figura 2C). Pode-se observar que esta espécie possui uma distribuição desbalanceada de indivíduos nas menores classes diamétricas, e que após 40 anos de sucessão florestal, provavelmente a população esteja perdendo a sua capacidade regenerativa e sendo excluída da área pela competição com espécies nativas. Aliado a este fator está a sua baixa frequência relativa e a forte concentração dos seus indivíduos no trecho da área de estudo que está mais sujeito a distúrbios por fatores antropogênicos. Estes dados podem nos fornecer importantes informações: a população está desbalanceada e com estoque reduzido de jovens, o que pode comprometer a sua capacidade de autorregeneração em longo prazo. Ou seja, o desenvolvimento das espécies da floresta e as condições geradas limitam a reinstalação do Pinus e, portanto, com o aumento da cobertura do solo com o passar do tempo haverá sua substituição.

A espécie exótica Syzygium jambos, por outro lado, apresenta grande estoque de indivíduos nas menores classes de diâmetro, mostrando uma tendência à franca estabilização na área. Originária da Ásia Tropical, esta espécie está amplamente disseminada pelo mundo e presente na Mata Atlântica brasileira (CARVALHO, 2005). Binggeli (2001) relata S. jambos como uma das principais espécies invasoras em florestas neotropicais, por sua facilidade de dispersão, tolerância ao sombreamento e raiz difusa e rasa, que dificultam o desenvolvimento de novas espécies de plantas no local de seu estabelecimento. A espécie ainda apresenta, em seu ciclo de vida, características que a torna uma invasora de sucesso, como rápida germinação, rápido crescimento das mudas e juvenis e precocidade reprodutiva, que em conjunto proporcionam um rápido crescimento de suas populações (CARVALHO, 2005).

O estudo de populações de espécies invasoras é importante para o conhecimento do papel funcional e de ocupação da espécie na comunidade, auxiliando no subsídio a projetos de conservação, manejo e combate à invasão. Na área estudada a espécie exótica $S$. jambos apresenta alto Valor de Importância (VI = 6,73\%) com a quase totalidade dos indivíduos (85\%) incluídos na classe de 5 a $10 \mathrm{~cm}$ de DAP (Figura 2D). Estes dados são indicativos de que a espécie se estabeleceu recentemente na área e que o processo de invasão está em fase inicial. Futuras análises na área através de monitoramento em longo prazo são necessárias para avaliar o grau de invasão de $S$. jambos e se isto tende a comprometer a estrutura e funcionamento da comunidade arbórea.

\section{Grupos ecológicos}

Com relação às síndromes de dispersão (Figura 3A), as maiores proporções de espécies e indivíduos zoocóricos seguiram o padrão encontrado para florestas tropicais estacionais semideciduais (NUNES et al., 2003), onde mais de $50 \%$ das espécies produzem frutos consumidos por aves e mamíferos (HOWE; SMALLWOOD, 1982). As espécies zoocóricas no fragmento têm sua importância, pois podem suportar diferentes grupos de animais, fornecendo recursos para a manutenção da fauna local. Isto possibilita a entrada de novas espécies no fragmento, dispersas pela fauna frequentadora do mesmo (GRIFFITH et al., 1996).

O número reduzido de espécies consideradas secundárias iniciais e tardias e a alta representatividade de pioneiras indicam que a área se encontra em um estágio inicial de sucessão (Figura 3B). Chazdon (2008) comenta que a elevada densidade de espécies pertencentes a grupos sucessionais iniciais é uma característica peculiar das florestas perturbadas. O passado de distúrbios da 
FIGURA 3: Distribuição das espécies e indivíduos em grupos ecológicos.

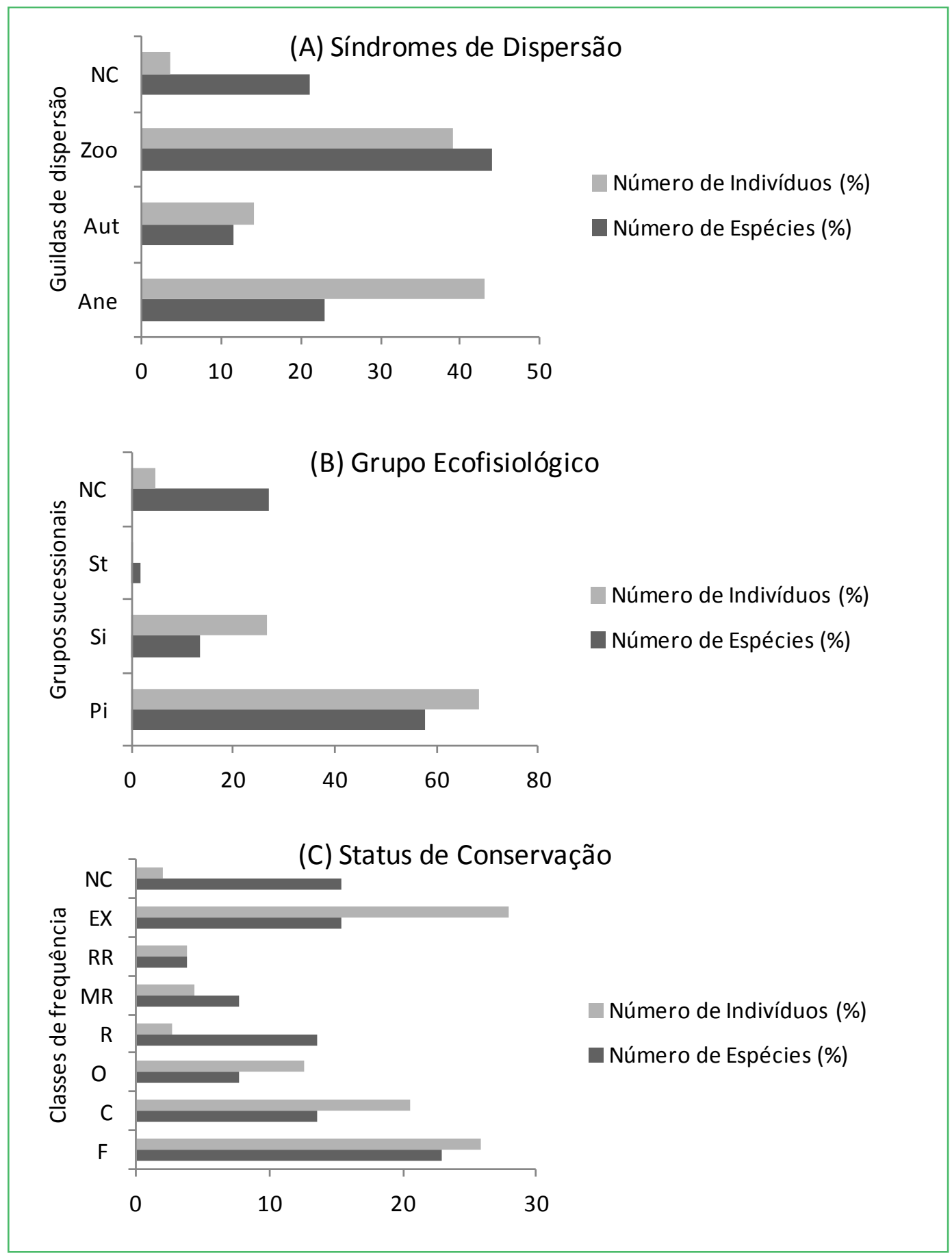

A - Grupo de síndromes de dispersão. B - Grupos ecofisiológicos. C - Grupos de status de conservação. Siglas: A - Síndrome de dispersão (Zoo: zoocórica; Ane: anemocórica; Aut: autocórica); B - grupo ecofisiológico (PI: pioneira; SI: secundária inicial; ST: secundária tardia; NC: não classificada); C - grupo de status de conservação (A: abundante; C: comum; F: frequente; O: ocasional; R: rara; MR: muito rara; RR: raríssima; EX: espécie exótica). 
área, anteriormente caracterizada como um pasto, e o pequeno tamanho do fragmento, que condiciona as espécies a crescerem sofrendo os efeitos de borda, podem ter interferido na alta proporção de espécies pioneiras e secundárias iniciais, e baixa presença de secundárias tardias.

A análise da classificação das espécies no grupo de status de conservação (Figura 3C) mostra que a comunidade é composta principalmente por espécies de distribuição "frequente" nas florestas de Minas Gerais. Apesar disso, também foram encontradas espécies com distribuição "rara" ou "muito rara" no estado, como as espécies Erythrina speciosa e Piptadenia gonoacantha. Este resultado não deve ser analisado apenas pelo seu caráter ecológico de distribuição geográfica restrita, mas também por serem espécies pouco amostradas no estado de Minas Gerais, que podem ocorrer com maior frequência em florestas de outros estados.

Em síntese, o fragmento florestal analisado apresenta um padrão ecológico peculiar, com forte dominância de duas espécies exóticas invasoras $(P$. elliottii e $S$. jambos), uma (P. elliottii) favorecida pela pressão de propágulos da arborização adjacente, mas que aparenta estar em processo de exclusão da comunidade, e a outra (S.jambos) pelas perturbações antrópicas no local e que se encontra em franca expansão na comunidade. A grande dominância exercida por $P$. elliottii e $S$. jambos certamente foi um fator que afetou negativamente a diversidade da área.

Como a regeneração de uma floresta caminha, geralmente, em direção à maior complexidade de formas, sendo influenciada diretamente pelo tempo decorrente desde a perturbação e pelo tipo de distúrbio, espera-se que a estratificação da floresta se torne mais complexa com a contribuição crescente de espécies de maior porte para o perfil da floresta, desde que as populações invasoras não interfiram negativamente nestes processos. O monitoramento da dinâmica desta vegetação, através da remedição destas parcelas permanentes e da mensuração em intervalos padronizados, será fundamental para avaliar o efetivo papel destas invasoras no funcionamento da comunidade e de que forma este remanescente se manterá florística e estruturalmente ao longo do tempo.

\section{Agradecimentos}

Agradecemos aos pesquisadores João Marcelo A. Braga (JBRJ), Daniel S. Pifano (IF-Goiano), Berenice C. Campos (UFJF) e colegas do herbário CESJ-UFJF pelo auxílio na identificação botânica; aos pesquisadores Arthur S. M. Valente (JBRJ) e Fátima R. G. Salimena (UFJF) pela revisão do trabalho realizado; aos alunos do Laboratório de Ecologia Vegetal (Departamento de Botânica/UFJF) pelo auxílio nos trabalhos de campo; à FAPEMIG pelo apoio financeiro (CRAAPQ 04438/10); à PROEX-UFJF pela bolsa de estudos concedida ao primeiro autor.

\section{Referências}

ALMEIDA, D. S.; SOUZA, A. L. Florística e estrutura de um fragmento de Floresta Atlântica, no município de Juiz de Fora, Minas Gerais. Revista Árvore, Viçosa, v. 21, n. 2, p. 221-230, 1997.

ALVEY, A. A. Promoting and preserving biodiversity in the urban forest. Urban Forestry \& Urban Greening, Frederiksberg, v. 5, p. 195-201, 2006.

APG. An update of the Angiosperm Phylogeny Group classification for the orders and families of flowering plants: APG III. Botanical Journal of the Linnean Society of London, London, v. 161, p. 105-121, 2009.

BINGGELI, P. The human dimensions of invasive woody plants. In: McNEELY, J. A. (Ed.). The great reshuffling: human dimensions of alien invasive species. Switzerland: IUCN, 2001. p. 145-159.

CARVALHO, F. A. Syzygium jambos (L.) Alston - uma invasora na Mata Atlântica? In: SIMPÓSIO BRASILEIRO DE ESPÉCIES EXÓTICAS E INVASORAS, I, 2005, Brasília. Resumos... Brasília: MMA. 2005. CD Rom.

CARVALHO, F. A.; NASCIMENTO, M. T. Estrutura diamétrica da comunidade e das principais populações arbóreas de um remanescente de Floresta Atlântica submontana (Silva Jardim-RJ, Brasil). Revista Árvore, Viçosa, v. 33, n. 2, p. 327-337, 2009.

CESAMA - COMPANHIA DE SANEAMENTO BÁSICO DE JUIZ DE FORA. Dados climáticos de Juiz de Fora, MG. 2010. Disponível em <www.cesama.com.br $>$. Acesso em: 10 novembro 2011.

CHAZDON, R. L. Chance and determinism in tropical forest sucession. In: CARSON, W. P.; SCHNITZER, S. A. (Ed.). Tropical forest community ecology. Chichester: Blackwell Publishing Ltd, 2008. p. 384-408.

FELFILI, J. M.; CARVALHO, F. A.; HAIDAR, R. F. Manual para o monitoramento de parcelas permanentes nos biomas Cerrado e Pantanal. Brasília: Universidade de Brasília, Departamento de Engenharia Florestal, 2005. 56 p.

GARCIA, P. O. Estrutura e composição do estrato arbóreo em diferentes trechos da reserva biológica municipal Santa 
Cândida, Juiz de Fora-MG. 2007. 104 f. Dissertação (Mestrado em Ecologia Aplicada ao Manejo e Conservação de Recursos Naturais.) - Universidade Federal de Juiz de Fora, Juiz de Fora. 2007.

GONZAGA, A. P. D.; OLIVEIRA-FILHO, A. T.; MACHADO, E. L. M.; HARGREAVES, P.; MACHADO, J. N. M. Diagnóstico florístico-estrutural do componente arbóreo da floresta da Serra de São José, Tiradentes, MG, Brasil. Acta Botanica Brasilica, Feira de Santana, v. 22, n. 2, p. 505-520, 2008.

GRIFFITH, J. J.; DIAS, L. E.; JUCKSCH, I. Recuperação de áreas degradadas usando vegetação nativa. Saneamento Ambiental, São Paulo, n. 37, p. 28-37, 1996.

HALPERN, C. B. Early successional patterns of forest species: interactions of life history traits and disturbance. Ecology, Stanford, v. 70, n. 3, p. 704-720, 1989.

HARPER, J. L., Population biology of plants. London: Academic Press, 1990. 892 p.

HOWE, H. F.; SMALLWOOD, J. Ecology of seed dispersal. Annual Review of Ecology, Evolution and Systematics, Palo Alto, v. 13, p. 201-228. 1982.

IBGE. Manual técnico da vegetação brasileira. Rio de Janeiro: Instituto Brasileiro de Geografia e Estatística, 2012. 271 p.

KENT, M.; COKER, P. Vegetation description and analysis. New York: John Wiley \& Sons, 1992. 365 p.

KÖPPEN, W. Das geographische System der Klimate - handbuch der klimatologie. Vol. 1. Part C. Berlim: Gebr. Bornträger Verlag, $1948.388 \mathrm{p}$.

KUEFFER, C. A global comparison of plant invasions on oceanic islands. Perspectives on Plant Ecology, Evolution And Systematics, Zurich, v. 12, p. 145-161, 2010.

LORENZI, H. Árvores brasileiras: manual de identificação e cultivo de plantas arbóreas nativas do Brasil. Nova Odessa: Plantarum, 1992. 352 p.

MAGURRAN, A. E. Measuring biological diversity. Oxford: Blackwell Science, 2004. 215 p.

MARANGON, L. C.; SOARES, J. J.; FELICIANO,A. L. P., BRANDÃO, C. F. L .S. Estrutura fitossociológica e classificação sucessional do componente arbóreo de uma floresta estacional semidecidual, no município de Viçosa, MG. Cerne, Lavras, v. 13, n. 2, p. 208-221, 2007.
MENON, T. A.; CARVALHO, F. A. Estrutura populacional de Pinus elliottii em áreas de regeneração florestal em Juiz de Fora, MG. Pesquisa Florestal Brasileira, Colombo, v. 32, n. 72, p. 367$372,2012$.

NUNES, Y. R. F.; MENDONÇA, A. V. R.; BOTEZELLI, L.; MACHADO, E. L. M.; OLIVEIRA-FILHO, A. T. Variações da fisionomia, diversidade e composição de guildas da comunidade arbórea em um fragmento de floresta semidecidual em Lavras, MG. Acta Botanica Brasílica, Feira de Santana, v. 17, n. 2, p. 213-229, 2003.

OLDEMAN, R. A. A. Dynamics in tropical rain forests. In: HOLMNIELSEN, L. B.; NIELSEN, I. C.; BALSLEV, H. (Ed.). Tropical forest: botanical dynamics, speciation and diversity. London: Academic Press, 1989. p. 3-21.

OLIVEIRA-FILHO, A. T.; FONTES, M. A. L. Patterns of floristic differentiation among Atlantic forests in south-eastern Brazil, and the influence of climate. Biotropica, Saint Louis, v. 32, n. 4, p. 793-810, 2000.

OLIVEIRA-FILHO, A. T.; SCOLFORO, J. R. S. Inventário florestal de Minas Gerais: espécies arbóreas da flora nativa. Lavras: Editora UFLA, 619 p. 2008.

PMJF. Anuário Estatístico de Juiz de Fora 2008. Prefeitura Municipal de Juiz de Fora. 2008. Disponível em <http://www. pjf.mg.gov.br/cidade/anuario_2008/index.html>. Acesso em: 19 novembro 2011.

ROCHA, G. C.; LATUF, M. O.; CARMO, L. F. Z. Mapeamento de riscos ambientais à escorregamentos na área urbana de Juiz de Fora, MG. Geografia, Londrina, v. 12, n. 1, p. 509-516, 2003.

RONDON-NETO, R. M.; BOTELHO, S. A.; FONTES, M. A. L.; DAVIDE, A. C.; FARIA, J. M. R. Estrutura e composição florística da comunidade arbustivo-arbórea de uma clareira de origem antrópica, em uma floresta estacional semidecídua montana, Lavras-MG, Brasil. Cerne, Lavras, v. 6, n. 2, p. 79-94, 2000.

UHL, C.; MURPHY, P. G. Composition, structure, and regeneration of a tierra firme forest in the Amazon basin of Venezuela. Tropical Ecology, Varanasi, v. 22, n. 2, p. 219-37, 1981.

VAN DER PIJL, L. Principles of dispersal in higher plants. 3 ed. Berlin: Springer-Verlag, 1982. 214 p. 